\title{
INTERPRETACIÓN DEL HABITAR UTILIZANDO DIMENSIONES SEMÁNTICAS ESCALAMIENTO MULTIDIMENSIONAL DEL ESPACIO SEMÁNTICO DE LA MACHIYA JAPONESA COMO BASE DE UN MÉTODO DE DISEÑO
}

DOI: https://doi.org/10.18861/ania.2019.9.2901

\author{
Dr. Arq. Fabián Jander
}

ORCID iD: http://orcid.org/0000-0002-8542-7876 


\section{DR. ARQ. FABIÁN JANDER}

Doctor en ingeniería, Departamento de arquitectura, Escuela de posgrado de ingeniería, Universidad de Kioto (Japón). Arquitecto, Escuela de Arquitectura y Diseño, Pontificia Universidad Católica de Valparaíso (Chile).

\section{COLABORADOR: DR. ARQ. TERUYUKI MONNAI}

Doctor en ingeniería, Escuela de Posgrado de Ingeniería, Universidad de Tokio (Japón). Master en Arquitectura, Escuela de Posgrado de Ingeniería, Universidad de Tokio (Japón). Arquitecto, Facultad de Ingeniería, Universidad de Kioto (Japón). Profesor del departamento de arquitectura de la Universidad de Arte de Osaka (Japón). Profesor Emérito de la Universidad de Kioto (Japón).

FECHA DE RECEPCIÓN: 31 de marzo de 2019.

FECHA DE ACEPTACIÓN: 21 de mayo de 2019.

REGISTRO BIBLIOGRÁFICO: JANDER, F. (2019). Interpretación del habitar utilizando dimensiones semánticas. Anales de Investigación en Arquitectura, 9 (1), 43-64. 


\section{RESUMEN}

Este estudio, consiste en extender con herramientas de análisis de datos nuestros estudios anteriores de las casas Machiya de Kioto, Japón, con el objetivo de crear la base de un método de diseño aplicable en arquitectura en general.

Empezaremos repasando algunos conceptos básicos utilizados en nuestro estudio, seguido por un breve análisis de la Machiya japonesa y su espacio semántico. Luego de presentar la casa Machiya, veremos algunos de los problemas a los que se enfrenta en la actualidad y como éstos se relacionan con un problema de identidad que la arquitectura enfrenta hoy en día, para luego a explicar los objetivos de esta investigación.

A continuación, presentaremos nuevas herramientas de análisis exploratorio de datos para analizar el espacio semántico, incluyendo agrupamientos y escalamiento multidimensional. Compararemos Sammon Mapping con Análisis de Componentes Principales, explorando sus usos potenciales para obtener información utilizable en el diseño de nuevos espacios arquitectónicos.

Veremos las posibilidades de aplicar las herramientas de análisis no solo para proyectos basados en arquitectura tradicional, sino que, para todo tipo de proyectos arquitectónicos y la creación de nuevos espacios.

Palabras clave: Análisis semántico, Análisis del espacio arquitectónico, Diseño arquitectónico, Arquitectura tradicional, Sammon Mapping, Análisis exploratorio de datos.

\section{ABSTRACT}

This study consists of extending the data analysis tools of our previous studies of Machiya houses in Kyoto, Japan, with the purpose of creating the foundations of a design method applicable in architecture in general.

We will start by reviewing some basic concepts used in our research, followed by a brief analysis of the Japanese Machiya and its semantic space. After presenting the Machiya house, we will review some of the problems it faces today and how these are related to an identity problem that architecture faces today, and then explain the research goals.

Next, we will present new exploratory data analysis tools to analyze the semantic space, including cluster analysis and multidimensional scaling. We will compare Sammon Mapping with Principal Component Analysis, exploring their potential uses to obtain usable information for the design of new architectural spaces.

We will see the possibilities of applying the tools for analysis not only for projects based on traditional architecture, but for all types of architectural projects and the creation of new spaces.

Key words: Architectural space analysis, Semantic analysis, Architectural design, Traditional architecture, Sammon Mapping, Exploratory data analysis. 


\section{INTRODUCCIÓN}

Cuando hablamos de arquitectura tradicional y arquitectura contemporánea, pareciera que la ruptura con estilos del pasado iniciada en el modernismo las separara en dos mundos diferentes. Sin embargo, todo tipo de arquitectura está ligada al habitar. Por lo tanto, resulta importante contar con metodologías utilizando esta continuidad, que puedan tratar tanto con contextos históricos tradicionales, como con la experimentación de nuevas posibilidades.

Siendo la arquitectura vernácula producto de siglos de experiencia y evolución acumulada, es de esperarse que desarrollemos metodologías para crear arquitectura basada en contextos tradicionales, que también se puedan utilizar al tratar con arquitectura vanguardista.

En este caso veremos a modo experimental, como el análisis semántico nos permitirá sumar puntos de vista para abordar el análisis del espacio y diseño en la arquitectura.

\section{EL ESPACIO SEMÁNTICO EN LA ARQUITECTURA}

En el modernismo se solía considerar que la forma obedecía a una función. Pero, así como Eco (1997), hace referencia a una connotación que acompaña a la función que la arquitectura denota, podemos observar en las ciudades de hoy en día como cuadrados y rectángulos representan la funcionalidad como una connotación más del espacio al que corresponden. Como explica Bonta (1979) gran parte del éxito del modernismo no fue ser funcional sino significar funcionalidad a modo de una cuestión valórica. La funcionalidad sola, no decide qué actividad se realiza en cuál espacio, sino que pasa a ser parte del lenguaje formal. Que un espacio sea "bueno" para algo sigue siendo una cuestión más bien de índole ética/estética, vale decir una interpretación del habitante, basada en sus valores culturales, que opera de modo similar a la interpretación en otras artes.

Neurocientíficos han encontrado algunas pistas sobre diferentes casos en los que experimentar la belleza en el arte está relacionado con nuestro sistema de recompensa en el cerebro, vinculado a nuestra evolución como especie (Dutton, 2009). Otras investigaciones, como la hipótesis de la sabana (africana), explican la preferencia por las representaciones de paisajes similares a la sabana es el producto de una preferencia que evolucionó en nuestros antepasados del Pleistoceno, donde esos paisajes eran un índice de seguridad y sustento (Chatterjee, 2014). Su belleza es ahora una especie de conocimiento tácito; un símbolo de supervivencia, al modo de lo "sublime" de Kant. Sin embargo, no necesitamos entender conscientemente las razones por las que disfrutamos estéticamente de los objetos. En el mismo sentido que Schellekens (2007) explica la relación entre lo bello y lo correcto, al hacer 
el habitante algo de un modo bello, estará estrechamente ligado a un modo correcto de hacer algo. Volviendo al caso modernista funcionalista, el habitante modernista supondrá hacer las cosas de bella o correcta manera, en cuanto las haga funcionalmente.

Pasando al caso de la estética japonesa, en el caso del elogio a la sombra (Tanizaki, 1977), la luminosidad juega un papel tan o más importante que la funcionalidad para el modernista en hacer las cosas de modo bello, que es a su vez un modo correcto de hacer las cosas dentro de su contexto cultural. Cabe destacar que ni para Tanizaki ni para el modernista existe un valor de luminosidad o funcionalidad absoluta. Para cada actividad pueden existir niveles que serán los "correctos" para dicha actividad ya sea de funcionalidad, luminosidad, privacidad, originalidad, seguridad y/o lo que corresponda dependiendo del contexto cultural del habitante.

De este modo, estas variables definen que actividad "significa" un espacio para su habitante de acuerdo a los valores que el espacio representa. Por ello llamamos estas variables "dimensiones semánticas". El espacio abstracto que se mide con estas dimensiones (que pueden ser muchas más de 3), lo llamaremos espacio semántico.

A modo burdo podremos ver que un habitante buscara espacios luminosos para leer, espacios oscuros para dormir o espacios privados para ducharse. Pero en realidad la situación es mucho más compleja y existen parámetros menos evidentes para diferentes actividades dependiendo del trasfondo cultural del habitante. Sería un error asumir que podemos ignorar o reducir estos parámetros a supuestos ideales universales de sentido común, ya que los valores culturales son bastante complejos y determinantes a la hora de habitar un espacio. Comprender el espacio semántico de algún tipo de arquitectura, nos permitirá a modo experimental ahondar más en el modo en que se habita e interpreta el espacio físico tridimensional.

\section{LA CASA TRADICIONAL MACHIYA}

La Machiya, literalmente casa de ciudad o casa tienda, es una vivienda urbana tradicional japonesa donde solían habitar familias dedicadas a actividades comerciales, incorporando tiendas y/o talleres en la vivienda. En nuestro estudio consideramos la Machiya de Kioto, como referencia para describirla nos guiaremos por Löfgren (2003) y el Kyoto Center for Community Collaboration (2009). Como arquitectura vernácula, corresponde a una tipología que fue evolucionando durante los siglos desde el periodo Heian (794 a 1185) hasta hoy, empezando a ser drásticamente reemplazada en el siglo 20 por edificios modernos, interrumpiendo su proceso evolutivo, lo que podría llevar a su extinción. 


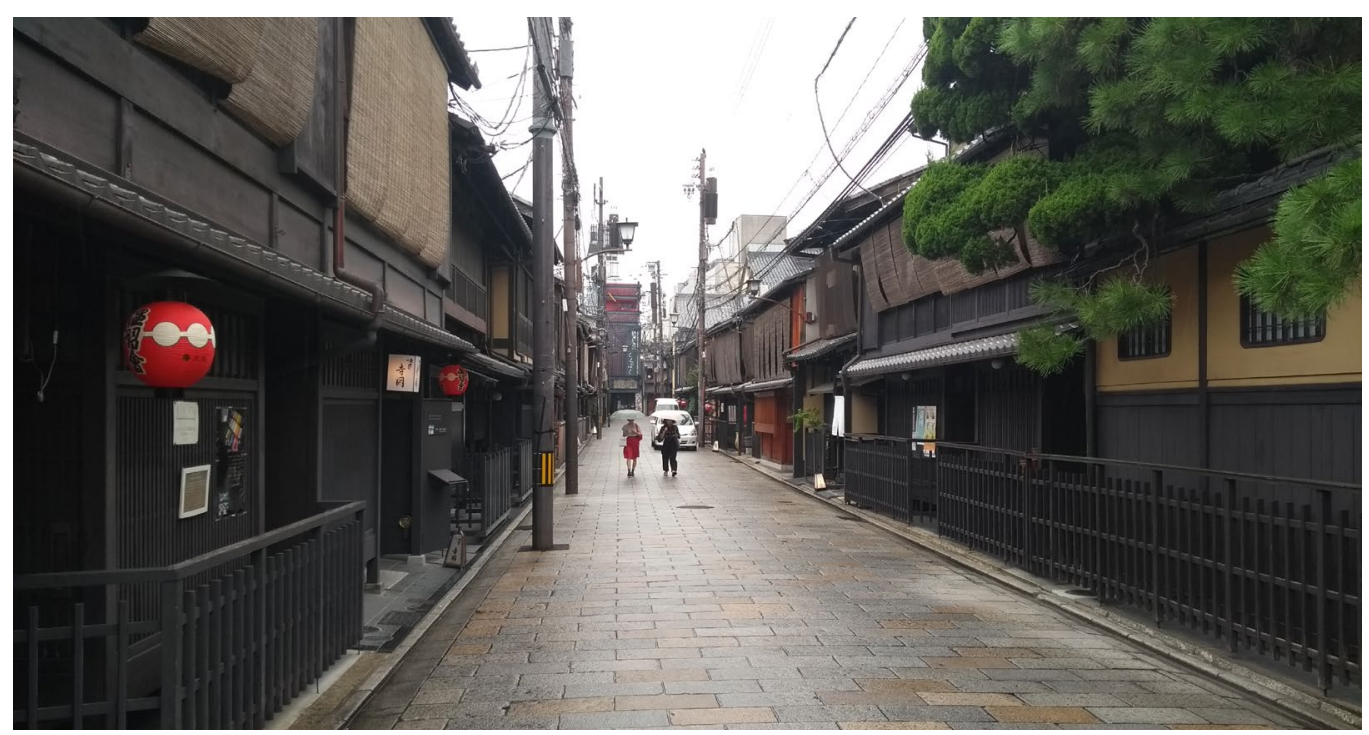

Figura 1.

Calle de Kioto en Gion en que se aprecia el paisaje urbano tradicional bien conservado, con casas Machiya a ambos lados.

La forma de la Machiya, en parte, se debe a fines prácticos. Dado a que existía un pago de impuesto proporcional al ancho de la fachada, las Machiyas suelen ser angostas y largas. La continuidad de las fachadas resultante conforma el típico paisaje urbano del antiguo Kioto.

La planta tipo de una Machiya como las que podemos encontrar en Kioto suelen tener un corredor doble altura que recorre la Planta desde la entrada hasta el fondo llamado Tooriniwa, que literalmente se podría traducir como patio de paso, que consiste en un espacio informal de carácter utilitario, que contrasta con los salones formales con piso de tatami y la tienda que se encuentran al lado opuesto.
Como se puede ver en la Figura 3, podemos ver una planta esquemática de Machiya que corresponde a un tipo básico de Machiya llamada “una fila-tres habitaciones" (松井, 2001), haciendo referencia a los 3 salones (6, $7,8)$ que se encuentran en una fila al lado del corredor "Tooriniwa" a la izquierda (2). En el segundo piso estarían los cuartos para dormir. Esta planta tipo de la Machiya la podemos dividir en 4 cuadrantes, en base al grado de privacidad y formalidad. Estos valores están intrínsicamente ligados a conceptos culturales japoneses, tales como "Hare" y "Ke" (extraordinario y ordinario), "Uchi" y "Soto" (interior y exterior, aplicado también a la familia), "Omote" y "Ura” (el frente y el reverso, donde el frente es la cara que se muestra). Las actividades extraordinarias, tales como ceremonias o recepción de clientes 


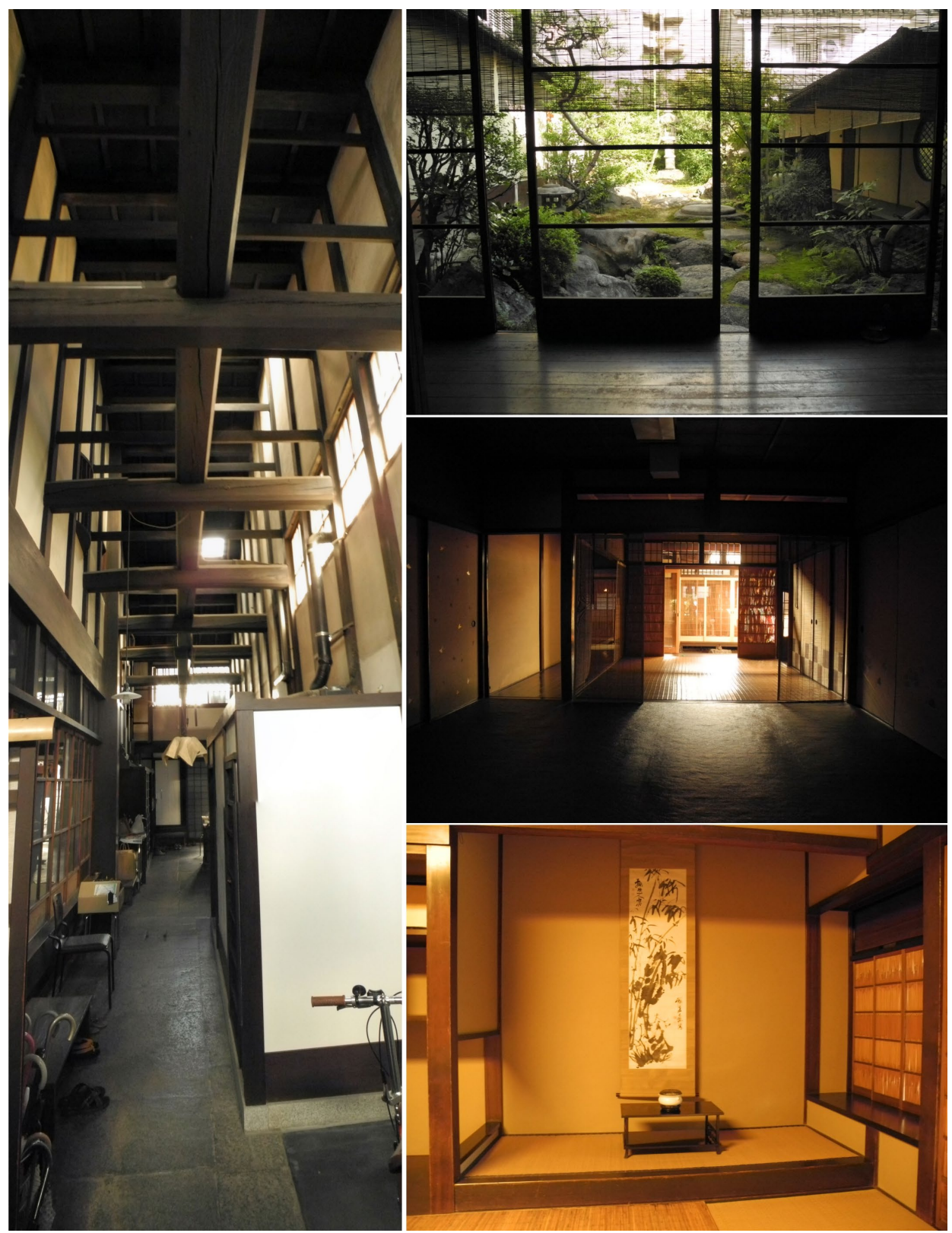

Figura 2.

Interior de una Machiya. A la izquierda se aprecia el Tooriniwa, que corresponde al lado informal de la casa. A la derecha, de arriba hacia abajo, ejemplos del lado formal de la casa: el jardín trasero (Okuniwa), vista de los salones hacia un patio interior (Tsuboniwa), un lienzo en la Tokonoma en el salón principal (Zashiki). 


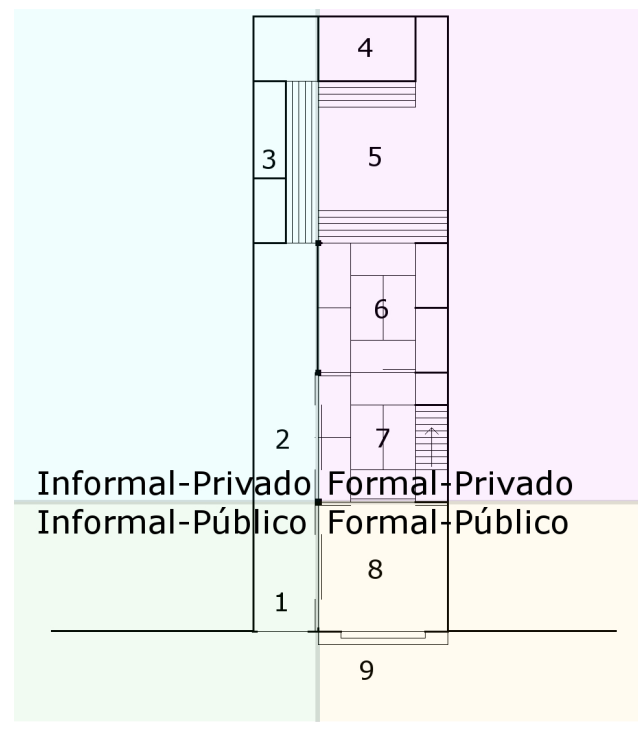

Figura 3.

Planta esquemática de una Machiya tipo, dividida en cuadrantes semánticos. 1: entrada (Genkan), 2: espacio utilitario a modo de corredor (Tooriniwa), 3: baños, 4: Bodega para elementos de valor presente en algunas Machiyas (Kura), 5: jardín trasero (Okuniwa), 6: salón principal con Tatami (Zashiki), 7: Salón central donde se suele comer (Daidoko, a veces también Nakanoma), 8: Tienda (Mise), 9: calle/exterior. (Elaboración propia) y huéspedes se llevan acabo en espacios formales, lo interno en espacios privados y el frente será lo formal-público (Omote) donde se puede encontrar la tienda, opuesto al reverso (Ura) que corresponde a lo informal-privado.

De esta forma la Machiya llegó a ser un excelente ejemplo de expresión cultural mediante la arquitectura.

En nuestro estudio (Autores, 2013a, 2013b) consideramos la privacidad, la formalidad y adicionalmente la luminosidad como descrita por Tanizaki (1977) y la naturaleza para analizar la Machiya. Estas 4 variables serán las "dimensiones semánticas" de las actividades que se llevan a cabo en el espacio semántico de la Machiya; para cada espacio hay un determinado nivel de privacidad, formalidad, luminosidad y naturaleza (conexión con naturaleza), que permiten al habitante interpretar cual actividad "significa" tal espacio dentro de la Machiya.

De este modo, habiendo muchas Machiyas diferentes, unas con más habitaciones, unas sin tienda, otras con talleres, algunas de una planta, la mayoría de 2 plantas y otras de 3; Machiyas con varios Patios interiores incluso algunas con un patio en el frente, etc., el habitante podrá "leer" las Machiyas y saber qué actividades corresponderán a cuál espacio en base a estas dimensiones. Incluso podremos detectar alteraciones contradictorias, como, por ejemplo, si instalásemos una cocina-comedor moderna en uno de los salones formales, ya que 


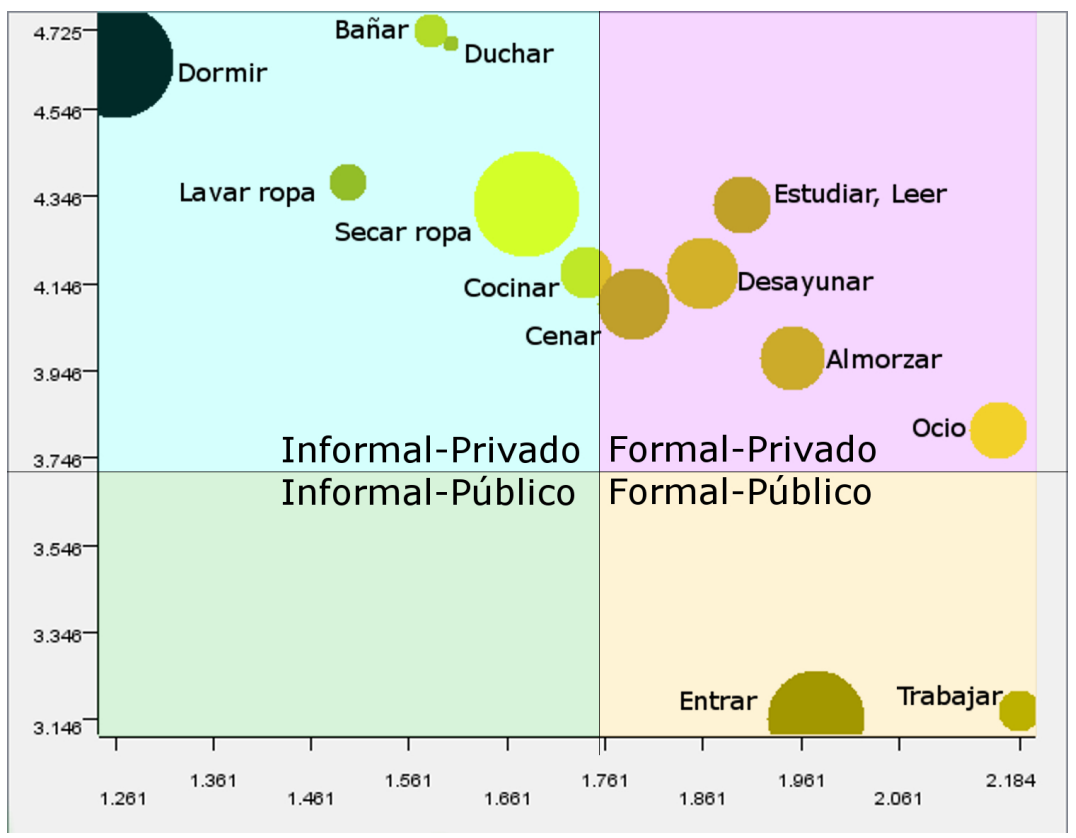

Figura 4.

presenta de izquierda a derecha la formalidad de menor a mayor. El exe-y representa de abajo hacia arriba la privacidad de menor a mayor. El color de los puntos representa la luminosidad, donde más claro (amarillo) indica mayor luminosidad. El tamaño de los puntos indica Naturaleza, indicando mayor tamaño un mayor grado de naturaleza. (Elaboración propia)

cocinar es considerada una actividad informal, o bien, el espacio formal no significa cocinar.

\section{ANÁLISIS SEMÁNTICO DE LA MACHIYA}

En nuestro estudio anterior (Autores, 2013a, 2013b) hemos visto como podemos diferenciar la Machiya de otras tipologías de vivienda en Kioto. Utilizando un cuestionario, elaboramos una base de datos con la información de 223 encuestados, basado en una lista de 13 actividades (entrar, desayunar, almorzar, cenar, cocinar, dormir, duchar(se), bañar(se), lavar ropa, secar ropa, ocio, estudiar/leer y trabajar) y las 4 dimensiones semánticas ya mencionadas (privacidad, formalidad, luminosidad y naturaleza). Entre las preguntas incluidas en el cuestionario se pedía evaluar cada dimensión semántica en una escala de 1 a 5 para cada actividad, tanto su situación actual como según su preferencia. También se pedía indicar si la vivienda evaluada era Machiya, otro tipo de casa, departamento o similar u otro tipo de vivienda. Con dicha información podemos calcular los valores promedio de privacidad, formalidad, luminosidad y naturaleza para cada una de las actividades según tipo de vivienda, así como los valores preferidos por los habitantes. 
Como resultado pudimos diferenciar las tipologías de vivienda y descubrimos que al graficar los valores promedio correspondientes a la Machiya, el resultado se correlaciona bastante con la planta tipo de una Machiya, con excepción de la actividad "dormir", que en la Machiya se ubicaría en la segunda planta y "entrar" que resultó ser una actividad formal (si bien la menos formal de las actividades públicas), mientras que la Machiya tipo tendría la entrada al lado informal (Figura 4). Mientras que los otros tipos de vivienda no se correlacionan con la Machiya.

Con los resultados obtenidos, pudimos ver que la identidad de la Machiya tal como la estudiamos con todos sus valores culturales, está correlacionada con la interpretación que su habitante reveló en la encuesta. Sin embargo, el espacio semántico analizado no corresponde a las casas Machiyas por el método empleado, sino porque se escogieron dimensiones semánticas correspondientes a los valores culturales que moldean el espacio de la Machiya, y porque se encuestó a habitantes de casas Machiya. Pero bien, se puede aplicar el método a otros habitantes, con otros valores culturales escogiendo las dimensiones semánticas correspondientes, que dependiendo del caso podrían ser muchas. En principio son los valores que para el habitante son considerados esenciales para habitar el espacio, y en el caso de tratar con habitantes cuyos valores culturales no han sido ampliamente estudiados, se pueden obtener mediante un cuestionario previo.
Tomando en cuenta que el mismo análisis se puede aplicar a otros tipos de vivienda o espacios arquitectónicos, y es aplicable a otras culturas con otros valores, no podemos descartar que nos enfrentemos a casos con un número mayor de dimensiones semánticas, de modo que resulta muy difícil visualizar y analizar los datos. Necesitaríamos medios adicionales para visualizar el espacio semántico para encontrar los patrones y relaciones que existen entre las actividades que se están analizando.

\section{LOS PROBLEMAS DE IDENTIDAD DE LA MACHIYA Y LA ARQUITECTURA EN LA ACTUALIDAD}

Actualmente en Kioto se puede ver que muchas casas Machiya han sido demolidas y reemplazadas por edificios modernos. Pero por otro lado hemos visto en secciones anteriores que la Machiya tiene un importante valor cultural y ha evolucionado durante siglos. En algunos barrios la Machiya tradicional como en Gion (Figura 01) podrá ser conservada como tal, pero en muchos casos como, por ejemplo, el de edificios de mayor altura, no es posible construir Machiyas de la misma forma. Por lo que en gran medida la Machiya tendrá que evolucionar o traspasarse a otras formas. Nuevas herramientas nos ayudarán para poder explorar alternativas para la evolución de la Machiya en el contexto contemporáneo. 
Por otro lado, el arquitecto suele quedar atrapado en las formas y materiales. Entonces, al tratar de crear nueva arquitectura basada en arquitectura tradicional, se suele imitar lo tradicional con formas y materiales, no necesariamente logrando el objetivo, resultando en más costos que beneficios.

Al enfocarnos en el habitante, cuya percepción e interpretación son las que deciden finalmente como se usará el espacio, podremos complementar nuestras herramientas de diseño, sumando nuevos puntos de vista, complementando los procesos creativos existentes.

Finalmente, a medida que el arquitecto genere menor impacto en como habitamos, se va volviendo menos relevante. Como ejemplo, cuando la industria de aparatos electrónicos puede llegar a tener mayor influencia a la hora de decidir como habitamos el espacio que un arquitecto. Muchos arquitectos se ven a veces relegados a vender imágenes, sin embargo, el verdadero potencial de la arquitectura es generar nuevas formas de habitar; dicha tarea tiene un lugar en la estructura social humana donde la arquitectura tiene más oportunidades de afectar a otros. Con lo cual, parafraseando a Wheeler (2006), una vez que algo pueda ejercer esta amplia gama de capacidades (para tener libertad semiótica), florecerá. En otras palabras, centrándose en el impacto visual de los edificios y no en el impacto que los edificios tienen en la vida humana, la arquitectura se debilita. El problema de identidad radica entonces en creer que la arquitectura trata de formas y no del habitar.

El análisis semántico puede ayudar a comprender la arquitectura (en este caso la Machiya) con un grado mayor de profundidad enfocándose en el habitar. Así se pueden tratar estos problemas a nivel del análisis, pero aún faltan herramientas para poder facilitar la creación de nueva arquitectura basada en un contexto cultural dado, que no necesariamente es la Machiya, sino que cualquier tipo de arquitectura.

Además, un problema que encontramos al trabajar con espacios semánticos con más de 3 dimensiones, es que a medida que aumenta el número de dimensiones, más difícil resulta encontrar relaciones entre las partes, encontrar patrones o agrupamientos, o siquiera visualizar dichos espacios abstractos.

\section{OBJETIVOS}

El objetivo principal de este estudio es extender el análisis semántico de modo que se facilite la extracción de patrones y relaciones para poder crear nueva arquitectura dentro de un contexto cultural dado. Como una herramienta complementaria para el diseño de espacios.

El análisis tendrá que ser capaz de procesar espacios semánticos de múltiples dimensiones, y el procedimiento tiene que ser matemáticamente fiable. 
Finalmente queremos lograr que se pueda utilizar la información obtenida como criterio de diseño, tanto para arquitectura tradicional como contemporánea.

\section{METODOLOGÍA}

Para facilitar en análisis y crear nuevas visualizaciones del espacio semántico proponemos usar métodos matemáticos para reducir el número de dimensiones necesarias para representar el espacio en 203 dimensiones que podamos visualizar fácilmente.

Como punto de partida tomaremos el espacio semántico de la Machiya de 4 dimensiones, conformado por puntos de datos que corresponden a las 13 actividades que analizamos anteriormente (Figura 04). Cada punto tendrá por lo tanto 4 dimensiones (formalidad, privacidad, luminosidad y naturaleza).

El mecanismo propuesto para reducir el número de dimensiones será "Sammon Mapping", un tipo de análisis exploratorio de datos que permite representar un espacio de $\mathrm{n}$ dimensiones en un espacio con un número menor de dimensiones. "Sammon Mapping” es un mecanismo no lineal, y que permite utilizar distancias en Manhattan, lo cuál es apropiado para puntos en un espacio con dimensiones que no interactúan entre ellas como lo harían en un espacio euclidiano. En el caso de Sammon
Mapping se busca minimizar las diferencias entre las correspondientes distancias entre puntos en el espacio original y los mismos puntos en el espacio con dimensiones reducidas, además de en lo posible no afectar la topología del espacio (Henderson, 2010). Como alternativa a comparar utilizaremos "Análisis de Componentes Principales" (ACP), que es otro método utilizado para reducir el número de dimensiones de un espacio transformando variables correlaciones entre las dimensiones originales en variables no correlacionadas (Peña, 2002).

Para comparar los resultados aplicaremos "Agrupamiento Jerárquico" para crear jerarquías de agrupamiento representadas en dendrogramas. Para el agrupamiento utilizaremos agrupamiento de enlace media o promedio con distancias en Manhattan. Aplicaremos el Agrupamiento Jerárquico a los datos correspondientes al espacio semántico de la Machiya de 4 dimensiones, el cuál utilizaremos de referencia, y luego aplicamos el mismo agrupamiento a los espacios reducidos a 2 o 3 dimensiones creados utilizando Sammon Mapping y ACP.

Luego de aplicar el agrupamiento descrito a cada uno de los espacios resultantes, compararemos los dendrogramas correspondientes a los espacios con menos dimensiones con nuestro dendrograma de referencia. Si los dendrogramas se mantienen similares, significa que las distancias entre los puntos se han 


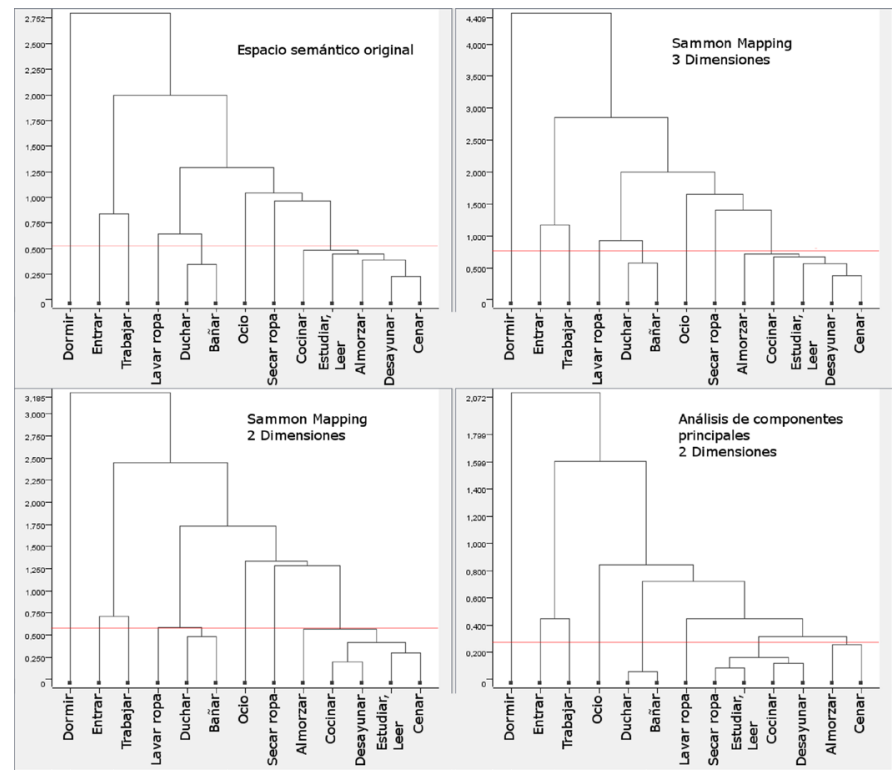

Figura 5.

Dendrogramas resultantes tras aplicar agrupamiento jerárquico al espacio semántico original, con reducción a 3 dimensiones utilizando Sammon Mapping, con reducción a 2 dimensiones utilizando Sammon Mapping y con reducción a 2 dimensiones utilizando análisis de componentes principales. La línea de corte de color rojo indica un corte para generar 8 grupos. (Elaboración propia)

mantenido proporcionales al espacio original, dado que los agrupamientos se generan a su vez a base de las distancias entre los puntos.

Para realizar los cálculos utilizaremos el software KNIME (versión 3.7.1, 2019), que tiene licencia GNU, facilitando el acceso para otros a las mismas herramientas de cálculo. Para los cálculos de Sammon Mapping, ACP, tanto como para los agrupamientos jerárquicos utilizamos los nodos correspondientes incluidos en el software. Para la representación de gráficos bidimensionales (diagramas de dispersión bidimensionales) se utilizará el nodo "Scatter Plot" incluido en el software, mientras que para los gráficos tridimensionales (diagramas de dispersión tridimensionales) se utilizará el nodo "2D/3D Scatterplot" de la extensión "Erlwood Knime Open Source Core" (versión 3.3.0, 2017). Es de esperarse que con ambos mecanismos (Sammon Mapping y ACP) se obtengan resultados adecuados para visualizar el espacio semántico de 4 dimensiones en 2 o 3 dimensiones manteniendo las principales relaciones entre los puntos, debido a que son métodos matemáticos creados para reducir la dimensionalidad de conjuntos de datos.

Teniendo visualizaciones en 2 o 3 dimensiones se podrá incluso optar por utilizar los gráficos resultantes como base para elaborar un plano 
Figura 6.

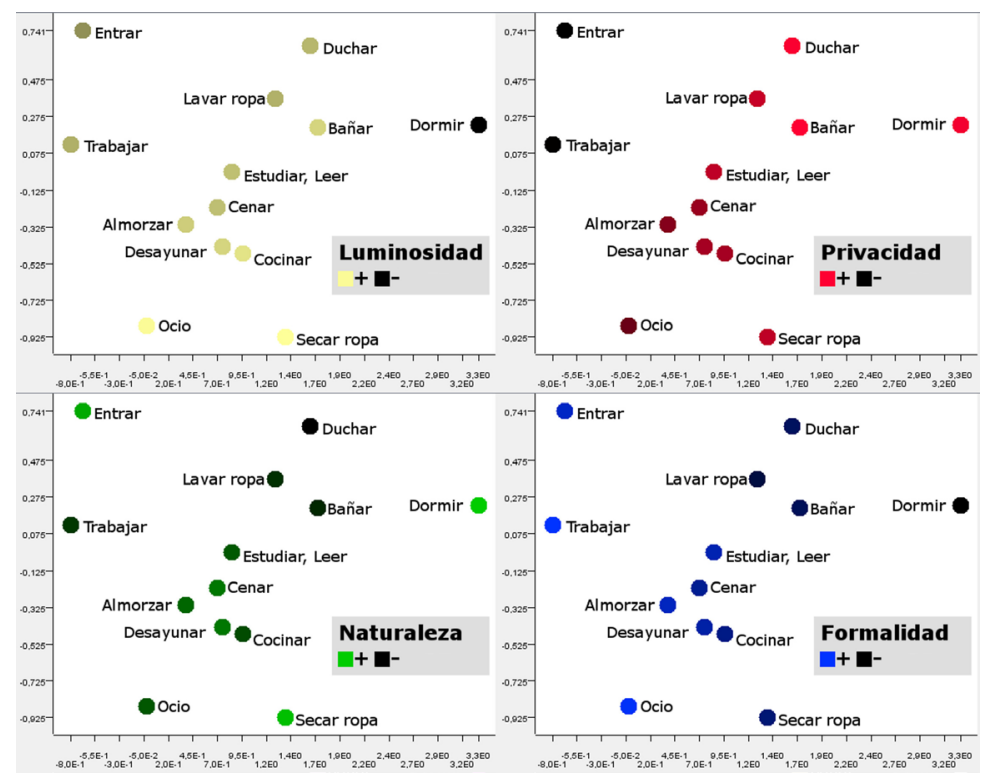

Espacio semántico de la Machiya proyectado en un diagrama de dispersión bidimensional utilizando Sammon Mapping repetido 4 veces, utilizando colores para mostrar cómo se distribuyen las 4 dimensiones originales (Luminosidad, Privacidad, Naturaleza y Formalidad) en el nuevo espacio bidimensional. (Elaboración propia)

para un diseño.

\section{REPRESENTACIÓN DEL ESPACIO SEMÁNTICO EN UN ESPACIO DE 203 DIMENSIONES}

Después de realizar los cálculos anteriormente descritos, pudimos verificar que utilizando Sammon Mapping los agrupamientos jerárquicos tienden a mantener mayor correlación con nuestra referencia correspondiente a los datos originales de 4 dimensiones. Sin embargo, los resultados obtenidos con el Análisis de Componentes Principales igualmente mantienen la estructura general del espacio original, como se aprecia en los dendrogramas en la figura 5.

Al utilizar Sammon Mapping, el agrupamiento jerárquico se mantiene similar al original, pudiéndose hacer un corte en los dendrogramas generando hasta 8 grupos con la misma jerarquía que en el espacio original, incluso al reducir el espacio a 2 dimensiones (Figura 05). Así se mantiene como grupo 1: "Dormir"; grupo 2: "Entrar"; grupo 3: "Trabajar"; grupo 4: "Lavar ropa”; grupo 5: "Duchar” y "Bañar”; grupo 6: "Ocio"; grupo 7: "Secar ropa"; grupo 8: "Almorzar", "Cocinar", "Estudiar, Leer", y "Cenar". Mientras que con el Análisis de Componentes Principales al generar 8 grupos ya hay algunas diferencias en el caso de 2 

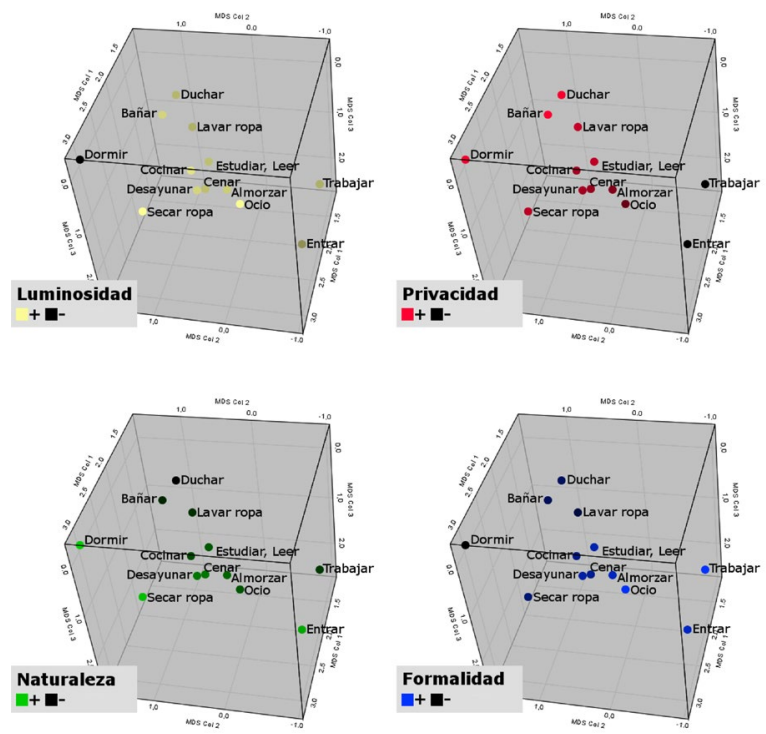

Figura 7.

Espacio semántico de la Machiya proyectado en un diagrama de dispersión tridimensional utilizando Sammon Mapping repetido 4 veces, utilizando colores para mostrar cómo se distribuyen las 4 dimensiones originales (Luminosidad, Privacidad, Naturaleza y Formalidad) en el nuevo espacio tridimensional. (Elaboración propia)

dimensiones, como se puede ver en la figura 05. En este caso, la actividad "Secar ropa" queda en el mismo grupo que "Estudiar, Leer", "Cocinar" y "Desayunar", mientras que "Almorzar" y "Cenar" se encuentran en un grupo aparte. Si bien los otros grupos se mantienen, hay también alteraciones a mayor jerarquía, como en el caso de "Ocio", que ya se configura como un grupo aparte a partir de la división en 4 grupos del dendrograma (Figura 5).

Obtener una correlación como la descrita, donde se mantiene la jerarquía del agrupamiento para hasta 8 grupos de un total de 13 actividades, es un nivel de correlación bastante elevado, ya que buscar agrupamientos donde el número de grupos se asemeja al número total de elementos es un despropósito, dado que nuestro objetivo es buscar relaciones o similitudes entre los elementos que justamente permitan agruparlos. En este sentido, el Análisis de Componentes Principales aún se podría utilizar como análisis exploratorio de datos para buscar nuevas alternativas de diseño con menor grado de correlación. Mientras que el resultado utilizando Sammon Mapping se recomendaría cuando se requiera trabajar de forma lo más fidedigna posible en el contexto original sin tener que copiar o imitar una obra existente. 
Debido a lo anterior, nos enfocaremos con mayor detalle a cómo utilizar Sammon Mapping para crear bases de diseño.

Comenzaremos con el espacio semántico de la Machiya proyectado en un espacio bidimensional.

Para poder comparar las 2 dimensiones nuevas con las originales, procedemos a graficar cada una de las 4 dimensiones semánticas originales sobre los diagramas de dispersión bidimensionales que produjimos con el método anteriormente explicado (Figura 6).

Analizando la figura 6 vemos que la privacidad se distribuye de forma opuesta a la formalidad, lo que refleja la diagonal entre formal-público e informal-privado, que también se aprecia en la figura 4, correspondiente a los conceptos Omote y Ura mencionados al describir las casas Machiya. Mientras que la luminosidad y la naturaleza no se distribuyen de forma lineal. En el caso de la luminosidad, la máxima se encuentra abajo donde se ubica el "Ocio" y "Secar ropa", y la mínima hacia la derecha donde se encuentra "Dormir". En cuanto a la naturaleza, ésta se concentra en polos opuestos; "Dormir" y “Secar la ropa” por un lado y "Entrar" por el otro.

Si bien nada de lo anterior contradice la información semántica que encontramos en la figura 04, la distribución de las actividades en la figura 6 es diferente. Lo que demuestra que podemos utilizar análisis exploratorio de datos para generar nuevas distribuciones de actividades semánticamente correlacionadas. Veremos a continuación que sucede al repetir el procedimiento, esta vez con 3 dimensiones Como podemos apreciar en la figura 7 , la distribución vuelve a cambiar, esta vez la naturaleza se distribuye de forma más lineal entre la actividad "Duchar" por un lado y las actividades "Entrar", "Secar ropa" y "Dormir" por el otro. Nótese que la actividad "Entrar" estaba al otro lado con respecto a "Secar ropa" y "Dormir" en el diagrama de dispersión bidimensional, pero aun así se mantiene el contexto semántico original. Mientras que los conceptos Omote y Ura siguen presentes en los opuestos formal-público e informal-privado.

Dado que se mantiene el contexto original, pero con distintas distribuciones, sumando la información obtenida de los agrupamientos jerárquicos y los diagramas de dispersión podemos utilizarla para crear un nuevo diseño basado en la Machiya tradicional de Kioto.

\section{APLICACIÓN DEL ANÁLISIS EXPLORATORIO DE DATOS SEMÁNTICOS EN EL DISEÑO}

Si quisiéramos elaborar un diseño nuevo, basado en la Machiya, podemos ocupar el análisis semántico de distintas formas. Podemos crear agrupamientos con algoritmos de agrupamiento como el agrupamiento jerárquico que utilizamos para agrupar actividades en espacios semánticamente similares. Dependiendo de la envergadura 
del proyecto podemos generar mayor o menor número de grupos. Podemos generar nuevas distribuciones en 2 o 3 dimensiones utilizando escalamiento multidimensional. Para proyectos más conservadores podemos utilizar Sammon Mapping y para buscar variantes con menor correlación con el espacio semántico original podemos utilizar Análisis de Componentes Principales. Dado que el espacio semántico es abstracto, podemos girar o distorsionar las distribuciones generadas manteniendo las propiedades topológicas para adaptarnos a distintos tipos de formas. Distribución de valores como luminosidad o naturaleza nos pueden ayudar a determinar orientación hacia luz solar, jardines $u$ otros elementos. En particular girar diagramas de dispersión tridimensionales puede ayudar a explorar posibilidades de distribución de espacios muy diversas.

Como ya se ha mencionado al final del análisis semántico de la Machiya, el método empleado representa a la Machiya tan solo por el uso de dimensiones semánticas correspondientes a los valores culturales que moldean el espacio de la Machiya, pero es aplicable a otros contextos semánticos. Así podemos utilizarlo para otras culturas incluyendo casos no relacionados con arquitectura tradicional.

Entre las muchas otras posibilidades de utilizar este tipo de análisis podemos incluir la experimentación con cambios de distribución que se generarían si a modo de hipótesis modificamos uno o más valores correspondientes a dimensiones semánticas de una o más actividades, ya que los métodos matemáticos utilizados generarían nuevas distribuciones correspondientes a tales modificaciones. De este modo también podemos simular cambios en valores culturales. Así como podemos representar escenarios hipotéticos, también es posible crear espacios semánticos personalizados, utilizando datos obtenidos de un habitante en particular, considerando sus propios valores culturales y actividades que quiera realizar. De este modo podemos realizar proyectos de arquitectura personalizados.

Al no existir limitaciones de escala ni de envergadura de proyectos a analizar, también podemos incluir en el análisis actividades que no necesariamente se realizarían dentro del proyecto, de modo que podemos integrar el proyecto dentro de un contexto mayor incluyendo actividades que se realizarían fuera de los límites del proyecto.

Al no existir un compromiso material ni formal en el análisis semántico, se mantiene gran libertad de acción en desarrollar un proyecto. La persona que emplea el método descrito, podrá mantener muchas de las prácticas de diseño que tenga; también es libre de elegir los materiales y formas o estilos que estime conveniente, siempre y cuando se respeten los valores designados para cada actividad. Así se podrán optimizar costos y adaptar el diseño a gusto sin sacrificar los valores esenciales del habitante. 


\section{CONCLUSIONES}

Pudimos comprobar que el análisis exploratorio de datos aplicado al análisis semántico sirve para generar directrices para crear nueva arquitectura semánticamente correlacionada con nuestro objeto de estudio, en este caso la Machiya.

Métodos como Sammon Mapping y Análisis de Componentes Principales ayudan a sugerir nuevas distribuciones. $Y$ en vez de haber solo un método que funcione, los distintos métodos serán utilizables para distintos propósitos, mostrando distintos grados de correlación, lo que se demostró utilizando agrupamientos jerárquicos.

Si bien los métodos empleados utilizan herramientas matemáticas, no se limita la creatividad, ya que, si bien se establecen relaciones entre las partes y se pueden describir según diversos valores, no se obliga a utilizar formas o materiales determinados, permitiendo optimizar costos.

Aún cuando el método se origina a base de una tipología de arquitectura tradicional específica, el nivel de abstracción permite que se pueda utilizar para todo tipo de arquitectura. Con la inclusión de escalamiento multidimensional se abren las puertas a espacios semánticos de mayor número de dimensiones semánticas. Incluso se podría utilizar para crear arquitectura personalizada o simular casos hipotéticos.
Al combinar distintos tipos de escalamiento multidimensional se pueden explorar múltiples posibilidades de distribución de modo que los métodos se complementan. Así no descartamos la experimentación con otros métodos de exploración de datos. Es más, los resultados sugieren que existe aún un potencial mucho mayor a descubrir experimentando con espacios semánticos.

Sumando los puntos anteriormente mencionados, podemos decir que, con el método propuesto, el arquitecto se enfoca netamente en el habitar, liberándose de la materialidad. Con una herramienta experimental que podrá utilizar para complementar sus modos de analizar y crear espacios. 


\section{BIBLIOGRAFÍA}

BONTA, J. P. (1979). Architecture and its interpretation: A study of expressive systems in architecture. London: Lund Humphries Publishers Ltd.

CHATTERJEE, A. (2014). The Aesthetic Brain: How We Evolved to Desire Beauty and Enjoy Art. Oxford: Oxford University Press.

DUTTON, D. (2009). The Art Instinct. Oxford: Oxford University Press.

ECO, U. (1997). Function and Sign: Semiotics and architecture. In N. Leach (Ed.) Rethinking Architecture: a reader in cultural theory (pp. 173-186) London an dNew York: Routledge.

ERLWOOD KNIME OPEN SOURCE CORE (versión 3.3.0) [extensión para el software Knime]. (2017). Surrey: Eli Lilly and Company Limited.

HENDERSON, P. (2010). Sammon Mapping. CVonline: On-Line Compendium of Computer Vision [Online]. Recuperado de http://homepages.inf.ed.ac.uk/rbf/CVonline/LOCAL_COPIES/AV0910/henderson.pdf

AUTORES, T. (2013a). Pragmatic Analysis of Modified Machiya Inhabitation - Culturally Friendly Design Method based on Machiya System of Kyoto (Part 2). Journal of Architecture and Planning (Transactions of AIJ), 78(690), 1771 1781 https://doi.org/10.3130/aija.78.1771

AUTORES, T. (2013b). Semantic Analysis of Machiya Inhabitation Context - Culturally Friendly Design Method based on Machiya System of Kyoto (Part 1). Journal of Architecture and Planning (Transactions of AIJ), 78(685), 573-
583 https://doi.org/10.3130/aija.78.573

KNIME (versión 3.7.1) [software]. (2019). Obtenido de http://www.knime.com

LÖFGREN, K. (2003). Machiya, architecture and history of the Kyoto town house (doctoral dissertation). KTH Royal Institute of Technology, Sockholm, Sweden.

KYOTO CENTER FOR COMMUNITY COLLABORATION. (2009). Machiya revival in Kyoto (2nd ed.). Kyoto: Mitsumura Suiko Shoin Publishing Co., Ltd.

MATSUI KAORU松井薰. (2001). Escuela de la Machiya de Kioto ¿qué es la Machiya? (Regeneración de la Machiya de Kioto Vol. 2) 京 町家塾 町家って何? (京町家再生 vol.2). Kioto: programa del fondo de la fundación de investigación para la regeneración de la Machiya de Kioto京都市: 京町家再生研究会助 成プログラム。

PEÑA, D. (2002). Componentes Principales. En Análisis De Datos Multivariantes (pp. 137178). Madrid: McGraw-Hill Interamericana de España, S.L.

SCHELLEKENS, E. (2007). Aesthetics \& Morality. London and New York: Continuum.

TANIZAKI, J. (1977). In Praise of Shadows. Sedgwick, Maine: Leete's Island Books.

WHEELER, W. (2006). Perfused with signs: biosemiotics and human sociality. In The Whole Creature: Complexity, Biosemiotics and the Evolution of Culture (pp. 106-130). London: Lawrence And Wishart Ltd. 


\section{FUENTE DE IMÁGENES}

Figuras 01 y 02. Fotos del autor. 